\title{
LA COLABORACIÓN EN LA CADENA DE SUMINISTROS: UNA REVISIÓN AL ESTADO DEL ARTE 1
}

\author{
COLLABORATION IN THE SUPPLY CHAIN: A STATE OF THE ART REVIEW \\ A COLABORAÇÃO NA CADEIA DE FORNECIMENTOS: UMA REVISÃO AO ESTADO DA ARTE \\ Bernardo Antonio Tamayo Arguello², Olga Rosana Romero Quiroga ${ }^{3}$, Mauricio Becerra Fernández ${ }^{4}$
}

Fecha de recibido: 05 de 2017 | Fecha de aprobado: Abril 22 de 2017

\section{Resumen}

El presente artículo busca identificar las tendencias, las características y los aspectos clave en torno al concepto de Colaboración en la Cadena de Suministros, lo cual permita proveer un estado del arte, teniendo presente que la información que se encuentra al respecto es limitada, dado que este concepto es relativamente nuevo en el ambiente logístico. La revisión y el análisis de publicaciones científicas fue dividida en tres fases: la primera, trata de la recolección de información por medio de la definición de artículos relevantes; la segunda, se enfoca en clasificar estos artículos mediante la identificación de tendencias; $y$, la tercera, busca plasmar los hallazgos en este documento para referencia en investigaciones futuras.

Palabras clave: administración de la Cadena de Suministro, Alianzas Estratégicas, Logística Colaborativa.

1 Artículo de revisión.

2 Ingeniero Industrial, Facultad de Ingeniería, Universidad Católica de Colombia. Program Supervisor, DHL Global Forwarding. Bogotá, Colombia. Correo electrónico: batamayo59@ucatolica.edu.co

3 Tecnóloga Industrial, Ingeniera de Producción, Especialista en Gestión de Proyectos de Ingeniería y Magíster en Ingeniería Industrial de la Universidad Distrital Francisco José de Caldas. Actualmente es candidata a doctor en Modelado en Política y Gestión Pública en la Universidad Jorge Tadeo Lozano de Bogotá, Colombia, y PhD. (c) in Model Based Public Planning, Policy Design and Management de la Universidad de Palermo, Italia. Se desempeña como Gerente de Gestión Documental en la empresa Suppla S.A. e investigadora en las áreas de logística, cadena de suministro, modelado y simulación. Correo electrónico: olga.romero@suppla.com

4 Tecnólogo Industrial, Ingeniero de Producción y Magíster en Ingeniería Industrial de la Universidad Distrital Francisco José de Caldas. Actualmente es candidato a doctor en Modelado en Política y Gestión Pública en la Universidad Jorge Tadeo Lozano de Bogotá, Colombia, y PhD. (c) in Model Based Public Planning, Policy Design and Management de la Universidad de Palermo, Italia. Se desempeña como profesor e investigador en las áreas de logística, producción, modelado y simulación en la Universidad Católica de Colombia. Director del Grupo de Investigación en Producción GIP. Correo electrónico: mbecerra@ucatolica.edu.co, mauriciobecerrafernandez@gmail.com 


\section{Abstract}

This article seeks to identify trends, characteristics and key aspects around the concept of collaboration in the supply chain. This will allow to provide a state of the art, bearing in mind that the information that is found on the topic is limited, since this concept is relatively new in the logistics field. The review and analysis of scientific publications was divided into three phases: the first one deals with the collection of information through the definition of relevant articles; the second focuses on classifying these articles through the identification of trends; and the third seeks to record the findings in this document for reference for future research.

Key Words: supply Chain Management, Strategic Alliances, Collaborative Logistics.

\section{Resumo}

Este artigo procura identificar as tendências, as características e os aspectos fundamentais em torno do conceito de Colaboração na Cadeia de Fornecimentos, o qual permita prover um estado da arte, tendo presente que a informação que se encontra ao respeito é limitada, dado que este conceito é relativamente novo no ambiente logístico. A revisão e a análise de publicações científicas foi dividida em três fases: a primeira trata da coleta de informação por meio da definição de artigos relevantes, a segunda foca-se em classificar estes artigos através da identificação de tendências e a terça procura plasmar os achados neste documento para referência em investigações futuras.

Palavras-chave: administração da Cadeia de Fornecimento, Alianças Estratégicas, Logística Colaborativa. 


\section{INTRODUCCIÓN}

Después de la Segunda Guerra Mundial las empresas han buscado aumentar su competitividad frente a los mercados, para así lograr ser sostenibles a través del tiempo. Para alcanzar este fin han desarrollado diferentes métodos, estrategias e iniciativas encaminadas a cubrir todo tipo de áreas (producción, logística, tecnología, calidad, etc.), tratando esencialmente de reducir los costos, aumentar la productividad, el reconocimiento y las utilidades. Teniendo en cuenta que no siempre la mejor estrategia a desarrollar es la reducción de costos, se han implementado métodos como la integración de la cadena de suministros, la producción Justo a Tiempo (JIT por sus siglas en inglés), la calidad total, entre otros. A lo largo de este trabajo de revisión se busca indagar acerca de un concepto relativamente nuevo en el campo logístico, la logística colaborativa. Esta propone romper una serie de paradigmas que se tienen acerca de la competencia entre empresas del mismo sector y establecer las bases para su introducción como una nueva filosofía empresarial.

\section{REVISIÓN Y DESARROLLO}

Al inicio de la revisión planteada se tenía la hipótesis de que las tendencias que se encontrarían variarían de acuerdo con el país de los autores y colaboradores en la construcción de estas referencias consultadas. También se había contemplado la posibilidad de clasificarlos de acuerdo a la industria donde se habían implementado y realizado las diferentes evaluaciones de estas prácticas. Sin embargo, una vez se dio inicio a la revisión de la información, se encontró que las hipótesis iniciales estaban lejos de ser correctas, ya que a medida que se avanzaba en la revisión, se identificaban tendencias y un método de clasificación que permitieron abarcar la información disponible (ver figura 1).

Figura 1. Tendencias de la información acerca de la colaboración en cadena de suministros

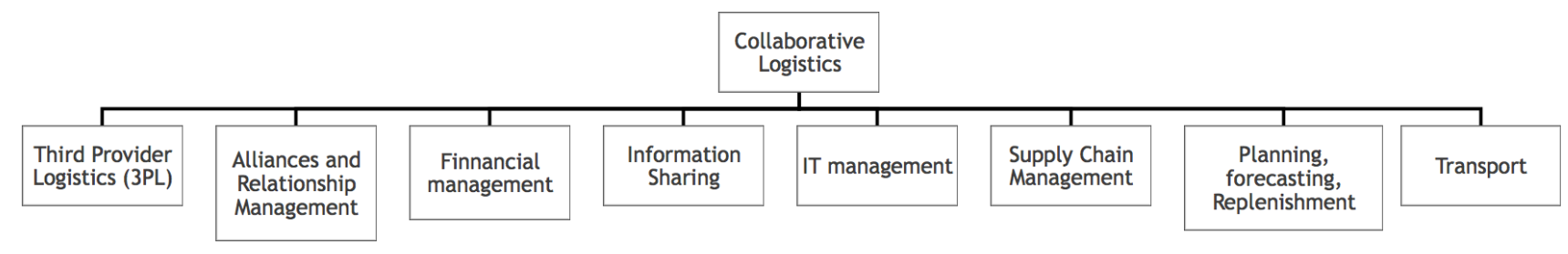

Fuente: elaboración propia.

De acuerdo con lo identificado anteriormente, se clasificaron los artículos consultados por el nivel de importancia que tienen para con el tema de la colaboración en las actividades logísticas, enfocándose en la colaboración en almacenamiento (ver tabla 1).

Tabla 1. Categorización de información

\begin{tabular}{|c|l|}
\hline NIVEL & \multicolumn{1}{c|}{ DESCRIPCIÓN } \\
\hline A1 & $\begin{array}{l}\text { Tiene que ver con el tema. Expresa claramente } \\
\text { la idea del artículo de revisión y contiene infor- } \\
\text { mación valiosa acerca del tema de interés. }\end{array}$ \\
\hline A2 & $\begin{array}{l}\text { Se acerca al tema. Si bien no expresa con cla- } \\
\text { ridad el tema del artículo de revisión, contiene } \\
\text { partes que pueden ayudar a su mejor entendi- } \\
\text { miento. }\end{array}$ \\
\hline A3 & $\begin{array}{l}\text { No se relaciona con el tema, a pesar de esto pue- } \\
\text { de contener elementos básicos que lleven a la } \\
\text { evolución que ha tenido el concepto. }\end{array}$ \\
\hline
\end{tabular}

Fuente: elaboración propia.
Conforme a lo descrito previamente, en la tabla 2 se encontrará un resumen de la clasificación realizada. Adicional a ello, se puede observar gráficamente este resumen en la figura 2.

Tabla 2. Clasificación según importancia en cuanto a la colaboración en almacenamiento

\begin{tabular}{|c|c|c|c|}
\hline NIVEL & CANTIDAD & PORCENTAJE & $\begin{array}{c}\text { PORCENTAJE } \\
\text { ACUMULADO }\end{array}$ \\
\hline A2 & 55 & $46 \%$ & $46 \%$ \\
\hline A3 & 33 & $28 \%$ & $74 \%$ \\
\hline A1 & 31 & $26 \%$ & $100 \%$ \\
\hline Total & 119 & $100 \%$ & \\
\hline
\end{tabular}

Fuente: elaboración propia. 
Figura 2. Clasificación según importancia en cuanto a la colaboración en almacenamiento

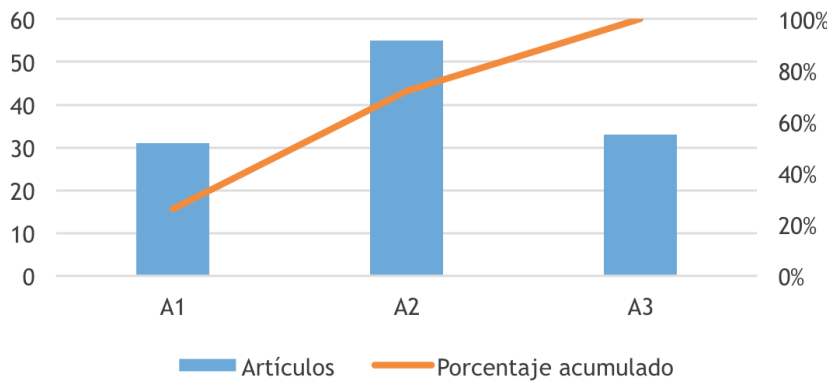

Fuente: elaboración propia.

De acuerdo con lo anterior, se encontró que la mayoría de los datos se concentran en los niveles $\mathrm{A} 2$ y A3, lo que lleva a deducir que la información disponible acerca de la colaboración en las actividades logísticas es muy limitada, especialmente en la temática principalmente abordada, ya que dentro de los artículos clasificados en el nivel A1, únicamente un artículo trata directamente de actividades relacionadas con el almacenamiento colaborativo. La distribución de dicha clase A1 se muestra en la tabla 3 y se puede observar de forma gráfica en la figura 3 .

Tabla 3. Distribución de artículos en la clase tipo A1

\begin{tabular}{|l|c|c|}
\multicolumn{1}{c|}{ SUBDIVISIÓN } & ARTÍCULOS & PORCENTAJE \\
\hline SCM & 14 & $45 \%$ \\
\hline $\begin{array}{l}\text { Alliances and Relationship } \\
\text { Management, }\end{array}$ & 8 & $26 \%$ \\
\hline IT Manageent & 2 & $6 \%$ \\
\hline $\begin{array}{l}\text { Planning, Forecasting, } \\
\text { Replenishment }\end{array}$ & 2 & $6 \%$ \\
\hline Transport & 2 & $6 \%$ \\
\hline 3PL & 1 & $3 \%$ \\
\hline Financial Management & 1 & $3 \%$ \\
\hline Information Sharing & 1 & $3 \%$ \\
\hline Total & 31 & $100 \%$ \\
\hline
\end{tabular}

Fuente: elaboración propia.
Figura 3. Distribución de artículos en la clase tipo A1

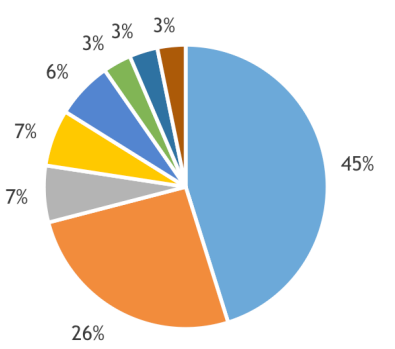

" SCM
" Alliances and Relationship Management
" IT Manageent
" Planning, Forecasting and Replenishment
" Transport
" 3PL
- Financial Management
- Information Sharing

Fuente: elaboración propia.

Con base en la clasificación y revisión mencionadas en las etapas iniciales, se procede a extractar los elementos que, a concepto de los autores, expresan mejor la idea de colaboración dentro de la cadena de suministros.

\section{Alianzas y gestión de relaciones (Alliances and relationship management)}

Si se quiere hablar de colaboración la primera idea que se evoca está relacionada con las alianzas entre los integrantes de la cadena para la consecución de objetivos. Recientemente el concepto de competencia ha tomado relevancia, describiendo una situación de negocios en la cual partes independientes cooperan entre sí y coordinan sus actividades, colaborando para conseguir sus objetivos, pero al mismo tiempo compitiendo entre sí y con otras firmas (Zineldin, 2004), lo que resulta ser muy aproximado a la idea de colaboración.

Cuando se habla de esta colaboración en la cadena de suministros, se contempla una estrategia para las empresas a la hora de crear una ventaja competitiva, haciendo que los costos se reduzcan, las mejoras en los ingresos sean notables y halla flexibilidad para hacer frente a la oferta y la demanda. Cabe resaltar que para lograr esto se requiere una gran cantidad de esfuerzo por parte de todos los miembros 
participantes, lo que facilita asegurar el logro de los beneficios potenciales mencionados.

Dentro de los requisitos que se deben cumplir para iniciar una relación colaborativa entre las empresas se encuentran los siguientes (Zineldin, 2004):

Dos o más individuos, grupos u organizaciones desean ser engranados en relación interactiva de intercambio.

Cada parte posee algo de valor que la otra parte desea.

Cada parte está dispuesta a entregar su algo de valor para recibir algo de valor que pertenece a la otra parte, en otras palabras, la relación se genera para que cada parte sea recompensada.

Cada parte es libre de aceptar o rechazar los términos y condiciones del intercambio que los dejará mejor (o al menos no peor) que antes del intercambio.

Las partes son libres de comunicarse e interactuar entre sí.

Las partes reconocen que las normas y valores éticos, interdependencia, compromiso y adaptación son cruciales para la creación, desarrollo y mejora positiva de una relación que sea sostenible a largo plazo.

Las partes pueden encontrar un balance positivo entre los pros y los contras de la relación.

Las alianzas y estrategias se han convertido en un tema crítico para la competitividad en el mundo, puesto que la consecución de objetivos y metas organizacionales requiere de la cooperación y la coordinación de las partes involucradas. Las alianzas son vistas ampliamente como acuerdos entre empresas para trabajar juntas y alcanzar un objetivo. Estos arreglos o acuerdos se logran por medio de fusiones, subcontratación y desarrollo de empresas de capital conjunto.

Dentro de las alianzas de colaboración se pueden encontrar dos tipos principales, que son las verticales y las horizontales. Las alianzas entre los competidores son conocidas como alianzas horizontales y se pueden distinguir de las alianzas verticales porque las firmas involucradas operan en etapas adyacentes a la cadena de suministros (Burgers, Hill, \& Kim, 1993). Por otra parte, las alianzas verticales son aquellas que unen a empresas en fases sucesivas de la cadena de valor, tratándose de acuerdos de colaboración entre proveedores y clientes (Africa, 2007).

Para Burgers, Hilly y Kim (1993) algunos de los incentivos para que las empresas inicien alianzas estratégicas son la poca certeza sobre la demanda y sobre la competencia. Las compañías cada vez se inclinan más a realizar alianzas con otras compañías para mejorar su desempeño en la cadena de suministros y su participación en el mercado, ya que estas alianzas les facultan para delegar algunas funciones a sus aliados y concentrarse en temas que les permitan mejorar su participación.

\section{Logística Tercerizada (Third Provider Logistics 3PL)}

El concepto de 3PL (Third Provider Logistics) tiene gran acogida en la medida que los productores y minoristas enfocan sus desarrollos en la cadena de suministros, recurriendo cada vez más a los $3 \mathrm{PL}$, no sólo para contar con un portafolio logístico más extenso, sino también para tener un cubrimiento geográfico mayor (Lieb \& Randall, 1999). Esto permite que las compañías se enfoquen en su actividad económica principal, delegando a estos 3PL las actividades logísticas en las que son expertos. Según Power, Sharafali y Bhakoo (2007) existen dos factores de los proveedores 3PL que influyen directamente en el desempeño de los clientes, tales son: las prioridades competitivas y los servicios ofrecidos. Estos conceptos se amplían a continuación.

\section{Prioridades competitivas}

Dentro de esta lista se destacan diez prioridades principales, como lo son: costos más bajos, mejor calidad, mayor variedad, más reacción, mayor flexibilidad, mejor seguridad, más innovación, paquetes de soluciones, soluciones personalizadas y total de soluciones. La combinación de servicios que proveen los 3PL es vista por los clientes como soporte a la flexibilidad, servicio al cliente y control de costos simultáneamente. Esta es una combinación potencialmente poderosa y puede explicar en parte el crecimiento de este sector.

\section{Servicios ofrecidos}

La combinación de un mejor control de inventario y la flexibilidad en la gestión de diversos productos, 
destacan la importancia de los $3 \mathrm{PL}$ para sus clientes (Power, Sharafali, \& Bhakoo, 2007). La literatura en 3PL presenta una variedad de perspectivas académicas y métodos de investigación, las cuales se pueden dividir en las siguientes tres categorías (Arroyo, Gaytan, \& De Boer, 2006):

La categoría A sugiere que los principales beneficios de los 3PL se encuentran en el ahorro de costos, la eficiencia operativa, la flexibilidad y el mejor servicio al cliente.

En la categoría B los trabajos se centran en el proceso de externalización de la logística y cómo se debe gestionar.

En la categoría $\mathrm{C}$ se incluyen los $3 \mathrm{PL}$ en un contexto más amplio en cuanto a las estrategias globales y la gestión de la cadena de suministro.

\section{Administración de la Cadena de Suministro (Supply Chain Management)}

Generalmente cada organización maneja de manera independiente las actividades de su cadena de suministros, existiendo planes no relacionados, tales como el reabastecimiento, los pronósticos, la programación de producción, etc. Los esfuerzos en este aspecto se enfocan en la planeación, la cual puede presentar falencias al no tomar en cuenta planes internos de las organizaciones y actividades que indudablemente impactarán el resultado de un plan en particular (Barrat, 2004).

Al tratar el tema de la colaboración en la cadena de suministro se genera una pregunta que es: ¿qué beneficios se pueden obtener de hacerlo? Antes de responder esta pregunta se deben considerar algunas de las siguientes preguntas (Barrat, 2004): ¿cuántas organizaciones sufren de una baja comunicación?, ¿cuántos planeadores conocen lo que está pasando a través de todas las partes de la organización, que se asocian o impactan el producto o actividad en la que se desarrolla?, ¿cuántas organizaciones conocen que sus propios procesos dejan aislados a sus proveedores o clientes?, si existen vacíos en el entendimiento de estos procesos, entonces, ¿cómo las organizaciones esperan mejorar sus procesos?

Estas preguntas deben ser analizadas para entender los beneficios que comprende el hecho de colaborar en la cadena de suministros. Por ejemplo, las mejoras en tecnologías de información pueden mejorar enormemente el desempeño de la cadena, como se puede presentar en los modelos de integración entre clientes y proveedores, en los que se busca conocer las necesidades de un cliente en tiempo real, de tal manera que se pueda reaccionar con mayor velocidad.

Como se muestra en la figura 4, existen dos maneras principales de colaboración, como ya se mencionó anteriormente. La primera es vertical, con proveedores y clientes, mientras que la segunda es horizontal, con competidores y no competidores compartiendo la capacidad de producción (Barrat, 2004).

Figura 4. Alcance General de La colaboración

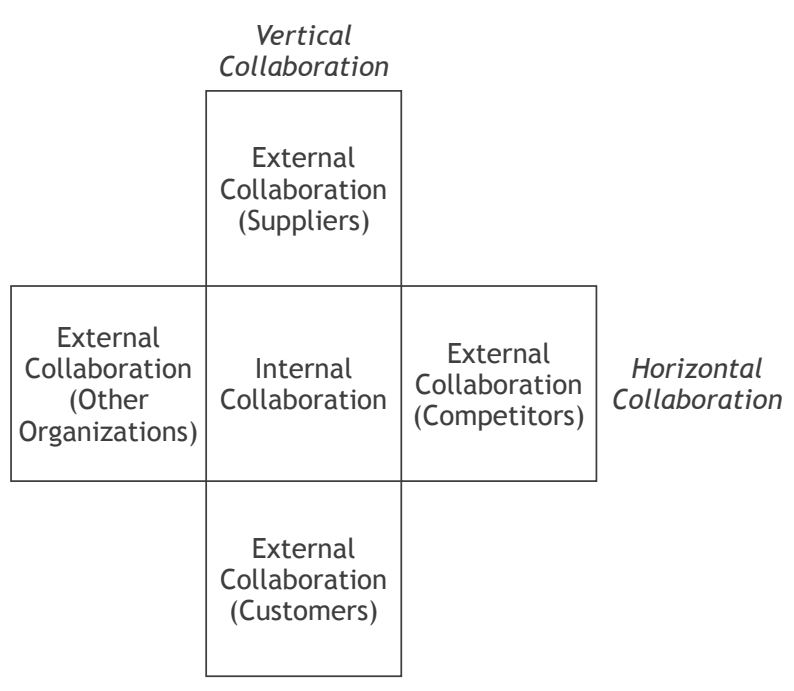

Fuente: Barrat, 2004.

Teniendo en cuenta lo anterior, se encuentran varias formas de colaboración, tales como CMR (Customer Relationship Management), CPFR (Collaborative Planning Forecast and Replenishment), vMl (Vendor Management Inventory), CRP (Continuous Replenishment Program), entre otras.

La tarea para las organizaciones se centra en identificar con quien se debe o no colaborar, ya que por desalentador que parezca, no es posible llevar a cabo la colaboración con todos los diversos proveedores, clientes y/o competidores. Es vital que antes de iniciar una propuesta de colaboración, las organizaciones involucradas evalúen si sus procesos y procedimientos son compatibles y, aún más importante, si sus objetivos corporativos lo son. 
Planificación de pronósticos y reabastecimiento (Planning forecasting and replenishment)

Dentro de los procesos de planificación se pueden ampliar los conceptos mencionados en torno a CPFR, VMI y CRP, de importancia en el marco del concepto de colaboración en la cadena de suministros.

Según Larsen, Thernoe y Claus (2003), existen tres clasificaciones que se le pueden dar a un CPFR de acuerdo al tipo de integración que se realice. CPFR básico, CPFR desarrollado y CPFR avanzado, como se muestra a continuación:

- CPFR básico: se involucran unos pocos procesos de negocios y se realiza una integración limitada entre los participantes. Los actores que han adoptado este enfoque CPFR usualmente seleccionan algunos procesos clave, en los que se realiza una colaboración con clientes y/o proveedores.

- CPFR desarrollado: se caracteriza por aumentar la integración en muchas áreas de la organización, cuando las partes que colaboran empiezan a coordinar datos e intercambio de información, haciendo acuerdos acerca de qué tipo de información van a intercambiar y cómo deberán responder a este intercambio.

- CPFR avanzado: difiere del anterior al llevar la colaboración más allá del mero intercambio de información. Adicional a dicho intercambio, se presenta un dialogo sincronizado entre las partes involucradas. Esto expande la colaboración a la sincronización de procesos, pronósticos, reabastecimiento y planeación.

Antes que una compañía pueda engranarse con otras en pronósticos colaborativos, debe establecer su propio proceso interno de estimación de la demanda mediante pronósticos. Para generar un entorno de colaboración en este aspecto, es necesario desarrollar procesos de pronostico consistentes, sistemáticos y apropiados que impacten positivamente el desempeño de la cadena de suministro, a través de disminuciones en costos operacionales, mejora del servicio al cliente, aumento en las ventas y reducciones en los inventarios (McCarthy \& Golicic, 2002).

Se pueden resaltar dos factores cuando se habla de colaboración en cuanto a pronósticos de la demanda: el primero se orienta a la adecuada definición de los procesos por parte de las compañías participantes y el segundo consiste en la implementación conjunta y sincronizada de los procesos asociados a la colaboración por parte de los actores involucrados.
En este orden de ideas, Mentzer (2005) caracteriza cuatro componentes que definen un buen proceso de pronóstico, como lo son la administración, los sistemas, las técnicas y la medición del desempeño.

\section{Transporte (Transport)}

Según Esper y Williams (2003) CPFR se plantea como una iniciativa para reducir riesgos y costos, mejorar el desempeño de la cadena y crear alianzas entre compañías, de lo que se deriva el concepto de стм (Collaborative Transportation Management).

El objetivo de стм es desarrollar relaciones colaborativas entre compradores, vendedores, transportistas y los 3PL para mejorar el servicio, la eficiencia y los costos asociados con el transporte y la entrega. Para la implementación de СтM es necesario considerar la aplicación de CPFR entre los involucrados, especialmente en los procesos de planificación del transporte. Así como en CPFR, en CTM los proveedores y clientes proveen información y colaboran con los transportadores para generar entregas eficientes (Esper \& Williams, 2003).

La gestión de transporte colaborativo ha sido nominada como el vínculo perdido de la ejecución de la cadena de suministros. En algunos casos se puede presentar poca habilidad para desarrollar efectivamente pronósticos de embarques, por tanto, los pronósticos de las órdenes realizadas mediante CPFR pueden resultar inexactos. En consecuencia, стм se considera un paso adicional y posterior a la creación de las órdenes mediante CPFR, ya que mientras que CPFR es inicialmente una relación entre el proveedor y el comprador, el concepto de стм añade al transportador dentro de la cadena de colaboradores.

\section{Administración Financiera (Financial Management)}

Por medio de la gestión financiera se busca desarrollar una gestión eficaz y eficiente del dinero, pero en la relación entre transportistas y proveedores se puede presentar baja satisfacción en el desempeño del otro con respecto al costo (Power, Sharafali, \& Bhakoo, 2007).

Stank, Keller y Closs (2001) analizaron la relación entre el rendimiento del servicio, la satisfacción del cliente, la lealtad del cliente y la cuota de mercado de la industria $3 \mathrm{PL}$, mediante la aplicación de encuestas a clientes. Las hipótesis planteadas fueron 
las siguientes; primero, existe una asociación significativa entre las prioridades competitivas y la contribución del 3PL para beneficio del cliente, segundo; existe una reciprocidad entre la oferta de servicios y el valor agregado del 3PL, y tres; existe una relación significativa entre el uso de los 3PL de las tecnologías y la contribución del $3 \mathrm{PL}$ para el rendimiento del cliente. Como conclusión se obtuvo que existe una asociación significativa entre las prioridades y la contribución del 3PL para el desempeño eficiente del cliente y se identificaron diez prioridades competitivas que deben tener los proveedores de servicios 3PL, las cuales fueron: menor costo, mayor calidad, mayor variedad, mayor sensibilidad, flexibilidad, mayor seguridad, innovación, soluciones empaquetadas, soluciones a la medida y soluciones globales.

\section{Suministro de información (Information sharing)}

Jeffrey muestra la colaboración entre los involucrados en una empresa de la industria automotriz en la cual se comparte información acerca de las operaciones y los costos de las mismas, accediendo adicionalmente a la información de otros proveedores, lo que permite tener elementos de evaluación y comparación, lo cual representa un reto en los niveles de satisfacción del cliente en la cadena de suministro colaborativa (Jeffrey, 1997). Cuando una empresa comparte información ampliamente, da una muestra de confianza para sus clientes, convirtiéndose esta confianza, en diversos escenarios, en la base de la colaboración (Dyer J. H., 1997).

Para el desarrollo de una relación cooperativa las relaciones industriales deben ser con frecuencia a largo plazo, puesto que esto implica un complejo patrón de interacción dentro de cada organización involucrada en dicha relación, ofreciendo sostenibilidad, dando muchas ventajas y oportunidades (Zineldin, 2004). Estas relaciones se puede esquematizar en la figura 5.
Figura 5. Modelo de colaboración entre empresas

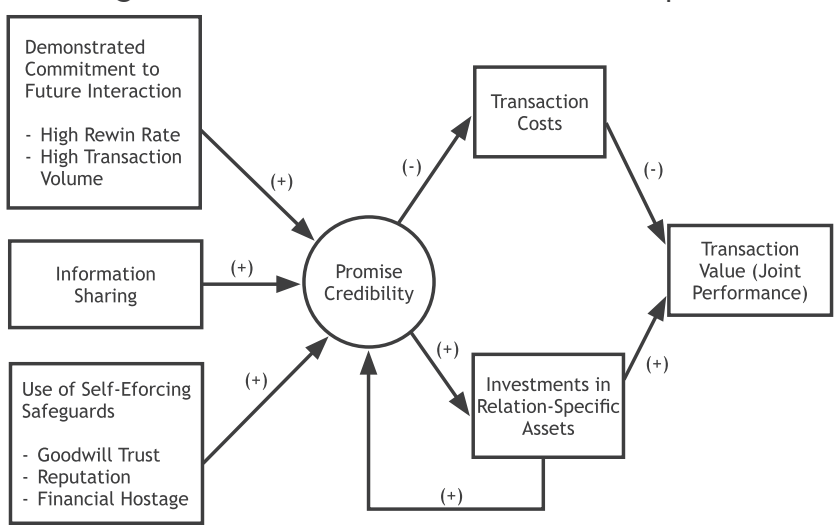

Fuente: Zineldin (2004).

\section{Administración de las tecnologías de la} información (IT Management)

Un aspecto relevante que ha evolucionado junto a las prácticas logísticas es la tecnología de la información involucrada (Information Technologies). Mediante el acceso a plataformas tecnológicas las empresas logran estar a la vanguardia en nuevas opciones logísticas, mejores rutas para transporte doméstico e internacional, entre otras.

Los avances en tecnologías de la información han generado cambios radicales en operaciones. Por ejemplo, hace una década era una práctica común que las compañías construyeran inventarios especulativos para acomodar estrategias de mercado, pero en la actualidad estas prácticas han venido siendo reemplazadas por estrategias de soporte logístico basadas en una respuesta oportuna. La disponibilidad de exactitud y la información a bajo costo a lo largo de la cadena de suministro, combinadas con la capacidad de compartir la información requerida, han guiado a arreglos logísticos estratégicos, como los propuestos por los modelos de respuesta rápida, JIT (Just In Time), de reabastecimiento continuo, de pronósticos y de planeación colaborativa (Bowersox \& Calantone, 1988). 
En el contexto de evolución y adopción de una scm, los productores y mayoristas están demandando un grado mayor de integración de los participantes de la cadena de suministro, en cuanto a los procesos de negocios a través de tecnologías de internet y de ICT (Information and Comunication Technologies). Como resultado, los 3PLs están devotamente incrementando la importancia de estas ICT en la administración de los negocios, convirtiéndose este concepto en uno de los mayores conductores de cambio, proponiendo nuevos retos a los proveedores logísticos (Evangelista \& Sweeney, 2006).

Impacto de las IT al interior de las organizaciones

Tradicionalmente, la centralización (o descentralización) ha sido vista como una consideración en el desempeño corporativo. Dentro de las firmas centralizadas, pocos gerentes de alto nivel retienen la mayoría de la autoridad en la toma de decisiones. Por otro lado, a los gerentes de nivel medio en estas firmas descentralizadas se les otorga empoderamiento para que tomen decisiones. Un nivel relativo de centralización-descentralización parece ser uno de los paradigmas tradicionales asociados a la selección de estrategias de colaboración. De manera creciente, los avances en las tecnologías de información, una amplia disponibilidad de estas a bajo costo y las comunicaciones de alto poder, están alterando dramáticamente la importancia que la estructura de las organizaciones y, más específicamente, la centralización juegan en la implementación de estrategias (Bowersox \& Calantone, 1988).

Esta revolución tecnológica ha dado lugar a nuevos servicios que las empresas pueden exigir u ofrecer para mejorar su desempeño logístico, entre los que se pueden destacar, como establecen Evangelista y Sweeney (2006), nuevos negocios en línea (e-business), llevando la venta de productos a este medio. Los inventarios y el almacenamiento de los productos se han llevado a este sistema, junto a cotizaciones y demás actividades; nuevas funciones con la introducción de intermediarios online, que reúnen tanto a proveedores como a compradores bajo sus páginas de internet. Tras estas nuevas alianzas ha surgido igualmente una nueva modalidad de negocio denominada 4PLS, que consiste en organizaciones que llevan un control cuidadoso de toda la cadena de suministros e integran todas sus actividades, involucrando en gran medida el concepto de colaboración en los procesos logísticos.

\section{FUENTES ANALIZADAS}

Con base en la revisión adelantada, se identifican por referencia los principales elementos contemplados en los artículos observados, como se muestra en la tabla 4.

Tabla 4. Resumen de fuentes analizadas

\begin{tabular}{|c|c|c|c|c|c|}
\hline FUENTE & AÑo & AUTOR & TÍTULO & $\begin{array}{l}\text { PRINCIPALES } \\
\text { ELEMENTOS } \\
\text { CONTEMPLADOS }\end{array}$ & REFERENCIA \\
\hline $\begin{array}{l}\text { Journal of Business } \\
\text { Logistics }\end{array}$ & 1987 & $\begin{array}{l}\text { Bowersox, Donald J.; } \\
\text { Daugherty, Patricia J. }\end{array}$ & $\begin{array}{l}\text { Emerging Patterns Of } \\
\text { Logistical Organization }\end{array}$ & $\begin{array}{l}\text { Supply Chain } \\
\text { Management }\end{array}$ & $\begin{array}{l}\text { (Bowersox \& } \\
\text { Daugherty, 1987) }\end{array}$ \\
\hline $\begin{array}{l}\text { Strategic } \\
\text { Management Journal }\end{array}$ & 1992 & $\begin{array}{l}\text { Ring, Peter Smith; Van } \\
\text { De Ven, Andrew H. }\end{array}$ & $\begin{array}{l}\text { Structuring Cooperative } \\
\text { Relationships Between } \\
\text { Organizations }\end{array}$ & Transactions Governance & $\begin{array}{l}\text { (Ring \& Van De } \\
\text { Ven, 1992) }\end{array}$ \\
\hline $\begin{array}{l}\text { Strategic } \\
\text { Management Journal }\end{array}$ & 1992 & $\begin{array}{l}\text { Smith Ring, Peter \& } \\
\text { Van De Ven, Andrew H. }\end{array}$ & $\begin{array}{l}\text { Structuring Cooperative } \\
\text { Relationships Between } \\
\text { Organizations }\end{array}$ & $\begin{array}{l}\text { alternativas de } \\
\text { cooperacion }\end{array}$ & $\begin{array}{l}\text { (Smith R. \& Van } \\
\text { De Ven, 1992) }\end{array}$ \\
\hline $\begin{array}{l}\text { Supply Chain } \\
\text { Management: An } \\
\text { International Journal }\end{array}$ & 1993 & $\begin{array}{l}\text { Burgers, Willem P; } \\
\text { Hill, Charles W. L; } \\
\text { Kim, W. Chan }\end{array}$ & $\begin{array}{l}\text { A theory of global } \\
\text { strategic alliances: The } \\
\text { case of the global auto } \\
\text { industry }\end{array}$ & Automative industry & $\begin{array}{l}\text { (Burgers, Hill, \& } \\
\text { Kim, 1993) }\end{array}$ \\
\hline Intereconomics & 1993 & Parkhe, Arvind & $\begin{array}{l}\text { Strategic Alliance } \\
\text { Structuring: A Game } \\
\text { Theoretic And } \\
\text { Transaction Cost } \\
\text { Examination Of Interfirm } \\
\text { Cooperation }\end{array}$ & $\begin{array}{l}\text { Dimensiones } \\
\text { estructurales, el } \\
\text { rendimiento }\end{array}$ & (Parkhe, 1993) \\
\hline $\begin{array}{l}\text { The Rand Journal of } \\
\text { Economics }\end{array}$ & 1993 & Spiegel, Yossef & $\begin{array}{l}\text { Horizontal } \\
\text { subcontracting }\end{array}$ & Relationship Management & (Spiegel, 1993) \\
\hline
\end{tabular}




\begin{tabular}{|c|c|c|c|c|c|}
\hline FUENTE & AÑO & AUTOR & TÍTULO & $\begin{array}{c}\text { PRINCIPALES } \\
\text { ELEMENTOS } \\
\text { CONTEMPLADOS }\end{array}$ & REFERENCIA \\
\hline $\begin{array}{l}\text { Academy of } \\
\text { Management. } \\
\text { The Academy of } \\
\text { Management Review }\end{array}$ & 1994 & $\begin{array}{l}\text { Ring, Peter Smith; Van } \\
\text { de Ven, Andrew H. }\end{array}$ & $\begin{array}{l}\text { Developmental } \\
\text { processes of cooperative } \\
\text { interorganizational } \\
\text { relationships }\end{array}$ & $\begin{array}{l}\text { Collaboration, } \\
\text { Information Sharing, } \\
\text { Benchmarking }\end{array}$ & $\begin{array}{l}\text { (Ring \& Van De } \\
\text { Ven, 1994) }\end{array}$ \\
\hline MIS Quarterly & 1996 & $\begin{array}{l}\text { Kumar, Kuldeep; van } \\
\text { Dissel, Han G. }\end{array}$ & $\begin{array}{l}\text { Sustainable } \\
\text { collaboration: Managing } \\
\text { conflict and cooperation } \\
\text { in interorganizational } \\
\text { systems }\end{array}$ & $\begin{array}{l}\text { Interorganizational } \\
\text { systems, sustainability, } \\
\text { organizational strategies, } \\
\text { collaborative use of IT/IS }\end{array}$ & $\begin{array}{l}\text { (Kumar \& Van } \\
\text { Dissel, 1996) }\end{array}$ \\
\hline $\begin{array}{l}\text { Strategic } \\
\text { Management Journal }\end{array}$ & 1997 & Dyer H. Jeffrey & $\begin{array}{l}\text { Effective interfirm } \\
\text { collaboration: how firms } \\
\text { minimize transaction } \\
\text { costs and maximize } \\
\text { transaction value }\end{array}$ & $\begin{array}{l}\text { Collaboration, } \\
\text { Information Sharing, } \\
\text { Benchmarking }\end{array}$ & (Dyer J. H., 1997) \\
\hline $\begin{array}{l}\text { Journal of Business } \\
\text { Logistics }\end{array}$ & 1998 & Joseph C. Andraski & $\begin{array}{l}\text { Leadership and the } \\
\text { realization of supply } \\
\text { chain collaboration }\end{array}$ & Leadership & (Andraski, 1998) \\
\hline $\begin{array}{l}\text { Academy of } \\
\text { Management. } \\
\text { The Academy of } \\
\text { Management Review }\end{array}$ & 1998 & $\begin{array}{l}\text { T. K. Das; Bing-Sheng } \\
\text { Teng }\end{array}$ & $\begin{array}{l}\text { Between Trust and } \\
\text { Control Developing } \\
\text { confidence in partner } \\
\text { cooperation in alliances }\end{array}$ & $\begin{array}{l}\text { Strategic Alliances, } \\
\text { Relationship Management }\end{array}$ & $\begin{array}{l}\text { (Das \& Teng, } \\
\text { 1998) }\end{array}$ \\
\hline $\begin{array}{l}\text { Academy of } \\
\text { Management. } \\
\text { The Academy of } \\
\text { Management Review }\end{array}$ & 1998 & $\begin{array}{l}\text { Jeffrey H. Dyer; Harbir } \\
\text { Singh }\end{array}$ & $\begin{array}{l}\text { The relational view: } \\
\text { Cooperative strategy } \\
\text { and sources of } \\
\text { interorganizational } \\
\text { competitive advantage }\end{array}$ & $\begin{array}{l}\text { Relationships } \\
\text { management }\end{array}$ & $\begin{array}{l}\text { (Dyer \& Harbir, } \\
\text { 1998) }\end{array}$ \\
\hline $\begin{array}{l}\text { The Journal of } \\
\text { Business and } \\
\text { Economic Studies }\end{array}$ & 1998 & Robert J. Hartwig & $\begin{array}{l}\text { Cooperation and } \\
\text { Competition: A } \\
\text { Comparative Review }\end{array}$ & $\begin{array}{l}\text { Collaboration, supply } \\
\text { chain evolution }\end{array}$ & (Hartwig, 1998) \\
\hline $\begin{array}{l}\text { Production } \\
\text { and Inventory } \\
\text { Management Journal }\end{array}$ & 1998 & Jan Holmstrom & $\begin{array}{l}\text { Implementing vendor- } \\
\text { managed inventory the } \\
\text { efficient way: A case } \\
\text { study of partnership in } \\
\text { the supply chain }\end{array}$ & $\begin{array}{l}\text { Relationship } \\
\text { Management, Supply } \\
\text { Chain Management }\end{array}$ & $\begin{array}{l}\text { (Holmstrom, } \\
\text { 1998) }\end{array}$ \\
\hline $\begin{array}{l}\text { The Journal of } \\
\text { Business \& Industrial } \\
\text { Marketing }\end{array}$ & 1999 & $\begin{array}{l}\text { Maria Bengtsson, Soren } \\
\text { Kock }\end{array}$ & $\begin{array}{l}\text { Cooperation and } \\
\text { competition in } \\
\text { relationships between } \\
\text { competitors in business } \\
\text { networks }\end{array}$ & $\begin{array}{l}\text { Relationships } \\
\text { management }\end{array}$ & $\begin{array}{l}\text { (Bengtsson \& } \\
\text { Kock, 1999) }\end{array}$ \\
\hline $\begin{array}{l}\text { Journal of Business } \\
\text { Logistics }\end{array}$ & 1999 & $\begin{array}{l}\text { Douglas M. } \\
\text { Lambert; Margaret A } \\
\text { Emmelhainz; John T } \\
\text { Gardner }\end{array}$ & $\begin{array}{l}\text { Building successful } \\
\text { logistics partnerships }\end{array}$ & $\begin{array}{l}\text { Strategic Alliances, } \\
\text { Relationship Management }\end{array}$ & $\begin{array}{l}\text { (Lambert, } \\
\text { Emmelhainz, \& } \\
\text { Gardner, 1999) }\end{array}$ \\
\hline Marketing Letters & 2000 & Aric Rindfleisch & $\begin{array}{l}\text { Organizational Trust and } \\
\text { Interfirm Cooperation: } \\
\text { An Examination of } \\
\text { Horizontal Versus } \\
\text { Vertical Alliances }\end{array}$ & $\begin{array}{l}\text { Interorganizational } \\
\text { Relationships, trust, } \\
\text { cooperation, alliances }\end{array}$ & (Aric, 2000) \\
\hline $\begin{array}{l}\text { International } \\
\text { Journal of Physical } \\
\text { Distribution } \\
\text { \& Logistics } \\
\text { Management }\end{array}$ & 2000 & $\begin{array}{l}\text { Hans-Christian Pfohl } \\
\text { and Hans Peter Buse. }\end{array}$ & $\begin{array}{l}\text { Inter-organizational } \\
\text { logistics systems in } \\
\text { flexible production } \\
\text { networks An } \\
\text { organizational } \\
\text { capabilities perspective }\end{array}$ & $\begin{array}{l}\text { Horizontal relationships, } \\
\text { lateral relationships, } \\
\text { circular relationships or } \\
\text { general reciprocal } \\
\text { dependencies }\end{array}$ & $\begin{array}{l}\text { (Pfohl \& Buse, } \\
2000 \text { ) }\end{array}$ \\
\hline $\begin{array}{l}\text { Journal of Supply } \\
\text { Chain Management }\end{array}$ & 2000 & $\begin{array}{l}\text { Judith M Whipple; } \\
\text { Robert Frankel }\end{array}$ & $\begin{array}{l}\text { Strategic alliance success } \\
\text { factors }\end{array}$ & $\begin{array}{l}\text { Relationships } \\
\text { management }\end{array}$ & $\begin{array}{l}\text { (Whipple \& } \\
\text { Frankel, 2000) }\end{array}$ \\
\hline $\begin{array}{l}\text { International } \\
\text { Journal of Logistics } \\
\text { Management }\end{array}$ & 2001 & $\begin{array}{l}\text { Douglas M. Lambert, } \\
\text { Terrace L. Pohlen }\end{array}$ & Supply chain metrics & $\begin{array}{l}\text { Cadena de suministro, } \\
\text { gestión de interfaz } \\
\text { entre el proveedor y la } \\
\text { integración del cliente }\end{array}$ & $\begin{array}{l}\text { (Lambert \& } \\
\text { Pohlen, 2001) }\end{array}$ \\
\hline
\end{tabular}




\begin{tabular}{|c|c|c|c|c|c|}
\hline FUENTE & AÑo & AUTOR & TÍTULO & $\begin{array}{l}\text { PRINCIPALES } \\
\text { ELEMENTOS } \\
\text { CONTEMPLADOS }\end{array}$ & REFERENCIA \\
\hline $\begin{array}{l}\text { Transportation } \\
\text { Journal }\end{array}$ & 2001 & $\begin{array}{l}\text { Paul D. Larson; Britta } \\
\text { Gammelgaard }\end{array}$ & $\begin{array}{l}\text { The logistics triad: } \\
\text { Survey and case study } \\
\text { results }\end{array}$ & $\begin{array}{l}\text { Coordination, supply } \\
\text { chain management, } \\
\text { logistics, information } \\
\text { exchange }\end{array}$ & $\begin{array}{l}\text { (Larson \& } \\
\text { Gammelgaard, } \\
\text { 2001) }\end{array}$ \\
\hline $\begin{array}{l}\text { Transportation } \\
\text { Journal }\end{array}$ & 2001 & Edward A. Morash & $\begin{array}{l}\text { Supply chain strategies, } \\
\text { capabilities, and } \\
\text { performance }\end{array}$ & $\begin{array}{l}\text { Business strategy, Supply } \\
\text { chain strategy, Supply } \\
\text { Chain capabilities and } \\
\text { combinations, Supply } \\
\text { chain performance }\end{array}$ & (Morash, 2001) \\
\hline $\begin{array}{l}\text { Transportation } \\
\text { Journal }\end{array}$ & 2001 & $\begin{array}{l}\text { Theodore P Stank; } \\
\text { Scott B Keller; David } \\
\text { J. Closs }\end{array}$ & $\begin{array}{l}\text { Performance benefits of } \\
\text { supply chain logistical } \\
\text { integration }\end{array}$ & scm Asses & $\begin{array}{l}\text { (Stank, Keller, \& } \\
\text { Closs, 2001) }\end{array}$ \\
\hline $\begin{array}{l}\text { International } \\
\text { Journal of Physical } \\
\text { Distribution } \\
\& \text { Logistics } \\
\text { Management }\end{array}$ & 2002 & $\begin{array}{l}\text { Teresa M. McCarthy; } \\
\text { Susan L. Golicic }\end{array}$ & $\begin{array}{l}\text { Implementing } \\
\text { collaborative forecasting } \\
\text { to improve supply chain } \\
\text { performance }\end{array}$ & $\begin{array}{l}\text { Planning, forecasting, } \\
\text { Replenishment } \\
\text { performance }\end{array}$ & $\begin{array}{l}\text { (McCarthy \& } \\
\text { Golicic, 2002) }\end{array}$ \\
\hline $\begin{array}{l}\text { Business Process } \\
\text { Management Journal }\end{array}$ & 2002 & $\begin{array}{l}\text { Togar M. Simatupang; } \\
\text { Alan C. Wright; } \\
\text { Ramaswami Sridharan }\end{array}$ & $\begin{array}{l}\text { The knowledge of } \\
\text { coordination for supply } \\
\text { chain integration }\end{array}$ & $\begin{array}{l}\text { Coordination, supply } \\
\text { chain management, } \\
\text { logistics, information } \\
\text { exchange. }\end{array}$ & $\begin{array}{l}\text { (Simatupang, } \\
\text { Wright, \& } \\
\text { Sridharan, 2002) }\end{array}$ \\
\hline Intereconomics & 2002 & Floris Vogelaar & $\begin{array}{l}\text { Modernisation of EC } \\
\text { competition law, } \\
\text { economy and horizontal } \\
\text { cooperation between } \\
\text { Undertakings }\end{array}$ & $\begin{array}{l}\text { Relationship } \\
\text { Management, Supply } \\
\text { Chain Management. }\end{array}$ & (Vogelaar, 2002) \\
\hline $\begin{array}{l}\text { Journal of Business } \\
\text { Logistics }\end{array}$ & 2003 & $\begin{array}{l}\text { Susan L. Golicic; } \\
\text { James H. Foggin; John } \\
\text { T. Mentzer }\end{array}$ & $\begin{array}{l}\text { Relationship magnitude } \\
\text { and its role in } \\
\text { interorganizational } \\
\text { relationship structure }\end{array}$ & $\begin{array}{l}\text { Relationships } \\
\text { management }\end{array}$ & $\begin{array}{l}\text { (Golicic, Foggin, } \\
\text { \& Mentzer, 2003) }\end{array}$ \\
\hline $\begin{array}{l}\text { International } \\
\text { Journal of Physical } \\
\text { Distribution } \\
\text { \& Logistics } \\
\text { Management }\end{array}$ & 2003 & $\begin{array}{l}\text { Tage Skjoett-Larsen; } \\
\text { Christian Thernoe; } \\
\text { Claus Andresen }\end{array}$ & $\begin{array}{l}\text { Supply chain } \\
\text { collaboration: } \\
\text { Theoretical perspectives } \\
\text { and empirical evidence }\end{array}$ & $\begin{array}{l}\text { scm Management, } \\
\text { Forecasting, Planning, } \\
\text { replenisment. }\end{array}$ & $\begin{array}{l}\text { (Larsen, Thernoe, } \\
\text { \& Claus, 2003) }\end{array}$ \\
\hline $\begin{array}{l}\text { Transportation } \\
\text { Journal }\end{array}$ & 2003 & $\begin{array}{l}\text { Terry L. Esper, Lisa R. } \\
\text { Williams }\end{array}$ & $\begin{array}{l}\text { The value of } \\
\text { Collaborative } \\
\text { Transportation } \\
\text { Management(Стм): Its } \\
\text { relationship to CPFR and } \\
\text { Information Technology }\end{array}$ & $\begin{array}{l}\text { tranporte, } \\
\text { logistica, cadena de } \\
\text { abastecimiento, СтM, CPFR. }\end{array}$ & $\begin{array}{l}\text { (Esper \& Williams, } \\
\text { 2003) }\end{array}$ \\
\hline $\begin{array}{l}\text { International } \\
\text { Journal of Physical } \\
\text { Distribution } \\
\text { \& Logistics } \\
\text { Management }\end{array}$ & 2003 & $\begin{array}{l}\text { Mosad Zineldin and } \\
\text { Torbjorn Bredenlow }\end{array}$ & $\begin{array}{l}\text { Strategic alliance: } \\
\text { synergies and challenges } \\
\text { A case of strategic } \\
\text { outsourcing relationship } \\
\text { "sour" }\end{array}$ & outsourcing & $\begin{array}{l}\text { (Zineldin \& } \\
\text { Bredenlow, 2003) }\end{array}$ \\
\hline $\begin{array}{l}\text { Supply Chain } \\
\text { Management }\end{array}$ & 2004 & Mark Barratt & $\begin{array}{l}\text { Understanding the } \\
\text { meaning of collaboration } \\
\text { in the supply chain }\end{array}$ & $\begin{array}{l}\text { Supply chain } \\
\text { management, Channel } \\
\text { relationships. }\end{array}$ & (Barrat, 2004) \\
\hline Internet Research & 2004 & $\begin{array}{l}\text { Dimitris Folinas; } \\
\text { Vicky Manthou; } \\
\text { Marianna Sigala; Maro } \\
\text { Vlachopoulou }\end{array}$ & $\begin{array}{l}\text { E-volution of a supply } \\
\text { chain: cases and best } \\
\text { practices }\end{array}$ & $\begin{array}{l}\text { Supply Chain } \\
\text { Management, Case } \\
\text { Studies, Electronic } \\
\text { commerce }\end{array}$ & $\begin{array}{l}\text { (Folinas, } \\
\text { Manthou, Sigala, } \\
\text { \& Vlachopoulou, } \\
\text { 2004) }\end{array}$ \\
\hline $\begin{array}{l}\text { Journal of Business } \\
\text { Logistics }\end{array}$ & 2004 & $\begin{array}{l}\text { Douglas M. Lambert, } \\
\text { A. Michael, Knemeyer } \\
\text { and John T. Gardner }\end{array}$ & $\begin{array}{l}\text { Supply Chain } \\
\text { Partnerships: Model } \\
\text { Validation And } \\
\text { Implementation }\end{array}$ & $\begin{array}{l}\text { Cadena de Suministro, } \\
\text { Modelo }\end{array}$ & $\begin{array}{l}\text { (Lambert \& } \\
\text { Knemeyer, 2004) }\end{array}$ \\
\hline $\begin{array}{l}\text { Benchmarking: An } \\
\text { international Journal }\end{array}$ & 2004 & $\begin{array}{l}\text { Togar M. Simatupang; } \\
\text { Ramaswami Sridharan }\end{array}$ & $\begin{array}{l}\text { Benchmarking supply } \\
\text { chain collaboration: An } \\
\text { empirical study }\end{array}$ & $\begin{array}{l}\text { Information Exchange, } \\
\text { Benchmarking, Supply } \\
\text { Chain Management }\end{array}$ & $\begin{array}{l}\text { (Simatupang \& } \\
\text { Sridharan, 2004) }\end{array}$ \\
\hline
\end{tabular}




\begin{tabular}{|c|c|c|c|c|c|}
\hline FUENTE & AÑO & AUTOR & TÍTULO & $\begin{array}{c}\text { PRINCIPALES } \\
\text { ELEMENTOS } \\
\text { CONTEMPLADOS }\end{array}$ & REFERENCIA \\
\hline $\begin{array}{l}\text { Supply Chain } \\
\text { Management: An } \\
\text { International Journal }\end{array}$ & 2004 & Togar M. Simatupang & $\begin{array}{l}\text { Applying the theory of } \\
\text { constraints to supply } \\
\text { chain collaboration }\end{array}$ & $\begin{array}{l}\text { Supply Chain } \\
\text { management, Constraint } \\
\text { handling, Performance } \\
\text { Measuremeny, Channel } \\
\text { Relationship }\end{array}$ & $\begin{array}{l}\text { (Simatupang T. } \\
\text { M., 2004) }\end{array}$ \\
\hline $\begin{array}{l}\text { Marketing } \\
\text { Intelligence \& } \\
\text { Planning }\end{array}$ & 2004 & Mosad Zineldin & $\begin{array}{l}\text { Co-opetition: the } \\
\text { organisation of the } \\
\text { future }\end{array}$ & $\begin{array}{l}\text { Collaboration, } \\
\text { Information Sharing }\end{array}$ & (Zineldin, 2004) \\
\hline $\begin{array}{l}\text { Transportation } \\
\text { Journal }\end{array}$ & 2005 & $\begin{array}{l}\text { Chad W. Autry; Stanley } \\
\text { E. Griffis }\end{array}$ & $\begin{array}{l}\text { A Social Anthropology } \\
\text { of Logistics Research: } \\
\text { Exploring Productivity } \\
\text { and Collaboration in an } \\
\text { emerging science }\end{array}$ & $\begin{array}{l}\text { scm, Collaboration, } \\
\text { Research }\end{array}$ & $\begin{array}{l}\text { (Chad \& Griffis, } \\
\text { 2005) }\end{array}$ \\
\hline $\begin{array}{l}\text { Supply Chain } \\
\text { Management }\end{array}$ & 2005 & Damien Power & $\begin{array}{l}\text { Supply chain } \\
\text { management integration } \\
\text { and implementation: a } \\
\text { literature review }\end{array}$ & $\begin{array}{l}\text { Integration, Supply } \\
\text { chain management, } \\
\text { management strategy }\end{array}$ & (Damien, 2005) \\
\hline $\begin{array}{l}\text { Journal of Business } \\
\text { Logistics }\end{array}$ & 2005 & $\begin{array}{l}\text { Susan L. Golicic; John } \\
\text { T. Mentzer }\end{array}$ & $\begin{array}{l}\text { Exploring The Drivers } \\
\text { Of Interorganizational } \\
\text { Relationship Magnitude }\end{array}$ & $\begin{array}{l}\text { Relationship magnitude, } \\
\text { Relationship structure, } \\
\text { Relationship type }\end{array}$ & $\begin{array}{l}\text { (Golicic \& } \\
\text { Mentzer, 2005) }\end{array}$ \\
\hline $\begin{array}{l}\text { The Journal of } \\
\text { Business \& Industrial } \\
\text { Marketing }\end{array}$ & 2005 & $\begin{array}{l}\text { Amjad Hadjikhani; } \\
\text { Peter Thilenius }\end{array}$ & $\begin{array}{l}\text { The impact of horizontal } \\
\text { and vertical connections } \\
\text { on relationships' } \\
\text { commitment and trust }\end{array}$ & $\begin{array}{l}\text { Channel relationship, } \\
\text { Organizational } \\
\text { structures, Horizontal } \\
\text { marketing, Vertical } \\
\text { marketing, trust. }\end{array}$ & $\begin{array}{l}\text { (Hadjikhani \& } \\
\text { Thilenius, 2005) }\end{array}$ \\
\hline $\begin{array}{l}\text { International } \\
\text { Journal of Physical } \\
\text { Distribution } \\
\text { \& Logistics } \\
\text { Management }\end{array}$ & 2005 & $\begin{array}{l}\text { Togar M. Simatupang; } \\
\text { Ramaswami Sridharan }\end{array}$ & $\begin{array}{l}\text { The collaboration index: } \\
\text { a measure for supply } \\
\text { chain collaboration }\end{array}$ & $\begin{array}{l}\text { Suppply Chain } \\
\text { Management, } \\
\text { Measurement, } \\
\text { Information strategy, } \\
\text { Incentive schemes, } \\
\text { Surveys }\end{array}$ & $\begin{array}{l}\text { (Simatupang \& } \\
\text { Sridharan, 2005) }\end{array}$ \\
\hline $\begin{array}{l}\text { Management } \\
\text { Decision }\end{array}$ & 2005 & $\begin{array}{l}\text { Emanuela Todeva, } \\
\text { David Knoke }\end{array}$ & $\begin{array}{l}\text { Strategic alliances and } \\
\text { models of collaboration }\end{array}$ & $\begin{array}{l}\text { Alianzas estratégicas, } \\
\text { estrategia corporativa, } \\
\text { las estructuras } \\
\text { organizativas }\end{array}$ & $\begin{array}{l}\text { (Todeva \& Knoke, } \\
\text { 2005) }\end{array}$ \\
\hline $\begin{array}{l}\text { Supply Chain } \\
\text { Management }\end{array}$ & 2005 & $\begin{array}{l}\text { Suhaiza Zailani and } \\
\text { Premkumar Rajagopal }\end{array}$ & $\begin{array}{l}\text { Supply chain integration } \\
\text { and performance:us } \\
\text { versus east asian } \\
\text { companies }\end{array}$ & scm Management & $\begin{array}{l}\text { (Zailani \& } \\
\text { Rajagopal, 2005) }\end{array}$ \\
\hline $\begin{array}{l}\text { The International } \\
\text { Journal of Logistics } \\
\text { Management }\end{array}$ & 2006 & $\begin{array}{l}\text { Pietro Evangelista, } \\
\text { Edward Sweeney }\end{array}$ & $\begin{array}{l}\text { Technology usage in the } \\
\text { supply chain: the case of } \\
\text { small 3PLs }\end{array}$ & $\begin{array}{l}\text { Supply chain } \\
\text { management, Italy, } \\
\text { Competitive advantage, } \\
\text { Communication } \\
\text { technologies }\end{array}$ & $\begin{array}{l}\text { (Evangelista \& } \\
\text { Sweeney, 2006) }\end{array}$ \\
\hline $\begin{array}{l}\text { Transportation } \\
\text { Journal }\end{array}$ & 2006 & $\begin{array}{l}\text { Michael J Maloni; Craig } \\
\text { R. Carter }\end{array}$ & $\begin{array}{l}\text { Opportunities for } \\
\text { Research in Third-Party } \\
\text { Logistics }\end{array}$ & $\begin{array}{l}\text { Supply Chain } \\
\text { management }\end{array}$ & $\begin{array}{l}\text { (Maloni \& Carter, } \\
\text { 2006) }\end{array}$ \\
\hline $\begin{array}{l}\text { The International } \\
\text { Journal of Logistics } \\
\text { Management }\end{array}$ & 2006 & $\begin{array}{l}\text { Samar K. } \\
\text { Mukhopadhyay, Robert } \\
\text { Setaputra }\end{array}$ & $\begin{array}{l}\text { The role of } 4 \mathrm{PL} \text { as } \\
\text { the reverse logistics } \\
\text { integrator Optimal } \\
\text { pricing and return } \\
\text { policies }\end{array}$ & $\begin{array}{l}\text { Supply chain } \\
\text { management, Italy, } \\
\text { Competitive advantage, } \\
\text { Communication } \\
\text { technologies }\end{array}$ & $\begin{array}{l}\text { (Mukhopadhyay \& } \\
\text { Setaputra, 2006) }\end{array}$ \\
\hline $\begin{array}{l}\text { International } \\
\text { Journal of Physical } \\
\text { Distribution } \\
\text { \& Logistics } \\
\text { Management }\end{array}$ & 2006 & Gunnar Stefansson & $\begin{array}{l}\text { Collaborative logistics } \\
\text { management and the } \\
\text { role of third-party } \\
\text { service providers }\end{array}$ & $\begin{array}{l}\text { Transportation, } \\
\text { Distribution, Third-Party } \\
\text { Vendors, Distribution } \\
\text { Management }\end{array}$ & $\begin{array}{l}\text { (Stefansson, } \\
\text { 2006) }\end{array}$ \\
\hline
\end{tabular}




\begin{tabular}{|c|c|c|c|c|c|}
\hline FUENTE & AÑo & AUTOR & TÍTULO & $\begin{array}{c}\text { PRINCIPALES } \\
\text { ELEMENTOS } \\
\text { CONTEMPLADOS }\end{array}$ & REFERENCIA \\
\hline $\begin{array}{l}\text { Journal of Cases } \\
\text { on Information } \\
\text { Technology }\end{array}$ & 2006 & $\begin{array}{l}\text { Yu Chung William } \\
\text { Wang; Shun Chuan Ho }\end{array}$ & $\begin{array}{l}\text { Information Systems } \\
\text { Dispatching in the Global } \\
\text { Environment, Acer, } \\
\text { A Case of Horizontal } \\
\text { inegration }\end{array}$ & $\begin{array}{l}\text { Driving foces(External } \\
\text { factors) } \\
\text { Driving foces(Internal } \\
\text { factors) }\end{array}$ & $\begin{array}{l}\text { (Wang \& Ho, } \\
\text { 2006) }\end{array}$ \\
\hline
\end{tabular}

Fuente: elaboración propia.

\section{CONCLUSIONES}

Teniendo en cuenta la revisión realizada se encuentran limitaciones en la literatura que aborda específicamente el tema de la colaboración en la cadena de suministros. Gran parte de esta literatura se centra en los proveedores logísticos, mostrando el gran impacto e influencia que tiene este actor en los resultados de la cadena. Esto hace que se incremente la tercerización de los procesos logísticos hacia compañías especializadas en el sector.

Un elemento relevante para la colaboración en la cadena de suministros es la relación de confianza que se crea entre las partes que la integran, por lo que ha de considerarse que las empresas deben generar lazos fuertes entre ellas para poder iniciar una relación de colaboración.

Mediante la conformación de redes en alianzas estratégicas colaborativas, las empresas involucradas pueden lograr una mejor asignación de los recursos para el desarrollo de sus procesos logísticos, tales como los recursos financieros y el aprovechamiento del capital humano. Esto se traduce en menores costos y mayores beneficios para los colaboradores y los clientes.

\section{REFERENCIAS}

Africa, A. (2007). Alianzas estrategicas: Opciones para el crecimiento de la empresa.

Andraski, J. C. (1998). Leadership and the realization of supply chain collaboration. Journal of Business Logistics.

Aric, R. (2000). Organizational Trust and Interfirm Cooperation: An Examination of Horizontal Versus Vertical Alliances. Marketing Letters.

Arroyo, P., Gaytan, J., \& De Boer, L. (2006). A survey of third party logistics in mexico and a comparison with reports on europe and Usa. International Journal of Operations \& Production Management, 241.

Barrat, M. (2004). Understanding the meaning of collaboration in the supply chain. Supply Chain Management, 30.

Bengtsson, M., \& Kock, S. (1999). Cooperation and competition in relationships between competitors in business networks. The Journal of Business \& Industrial Marketing.

Bowersox, D. J., \& Calantone, R. J. (1988). Executive insights: global logistics. Journal of Business Logistics, 83.

Bowersox, D. J., \& Daugherty, P. J. (1987). Emerging Patterns Of Logistical Organization. Journal of Business Logistics, 46.

Burgers, W. P., Hill, C. W., \& Kim, C. W. (1993). A Theory of of global strategicalliances: The case of the Global Auto Industry. Supply Chain Management: An International Journal, 419.

Chad, W. A., \& Griffis, S. E. (2005). A Social Anthropology of Logistics Research: Exploring Productivity and Collaboration in an emerging science. Transportation Journal.

Damien, P. (2005). Supply chain management integration and implementation: a literature review. Supply Chain Management.

Das, T. K., \& Teng, B.-S. (1998). Between Trust and Control Developing confidence in partner cooperation in alliances. Academy of Management. The Academy of Management Review.

Dyer, J. H. (1997). Effective interfirm collaboration: how firms minimize transaction costs and 
maximize transaction value. Strategic Management Journal.

Dyer, J., \& Harbir, S. (1998). The relational view: cooperative strategy and sours of interorganizacional. Academy of Management. The Academy of Management Review, 660.

Esper, T. L., \& Williams, L. R. (2003). The value of Collaborative Transportation Management. Transportation Journal, 55.

Evangelista, P., \& Sweeney, E. (2006). Technology usage in the supply chain: the case of small 3PLs. The International Journal of Logistics Management, 55.

Folinas, D., Manthou, V., Sigala, M., \& Vlachopoulou, M. (2004). E-volution of a supply chain: cases and best practices. Internet Research, 274.

Golicic, S. L., \& Mentzer, J. T. (2005). Exploring The Driver Of Interorganizationl Relationship Magnitude. Journal of Business Logistics.

Golicic, S. L., Foggin, J. H., \& Mentzer, J. T. (2003). Relationship magnitude and its role in interorganizational relationship structure. Journal of Business Logistics.

Hadjikhani, A., \& Thilenius, P. (2005). The impact of horizontal and vertical connections on relationships' commitment and trust. The Journal of Business \& Industrial Marketing.

Hartwig, R. J. (1998). Cooperation and Competition: A Comparative Review. The Journal of Business and Economic Studies.

Holmstrom, J. (1998). Implementing vendor-managed inventory the efficient way: A case study of partnership in the supply chain. Production and Inventory Management Journal, 1.

Jeffrey, D. (1997). Effective interfirm collaboration: how firms minimize transaction costs and maximize transaction value. Strategic Management Journal, 551.

Kumar, K., \& Van Dissel, H. G. (1996). Sustainable collaboration: Managing conflict and cooperation in interorganizational systems. M/S Quarterly, 279-300.
Lambert, D. M., \& Knemeyer, A. M. (2004). Supply Chain Partnerships: Model Validation And Implementation. Journal of Business Logistics.

Lambert, D. M., \& Pohlen, T. L. (2001). Supply chain metrics. International Journal of Logistics Management.

Lambert, D., Emmelhainz, M. A., \& Gardner, J. T. (1999). Building successful logistics partnerships. Journal of Business Logistics.

Larsen, T. S., Thernoe, C., \& Claus, A. (2003). Supply chain collaboration: Theoretical perspectives and empirical evidence. International Journal of Physical Distribution \& Logistics Management, 531-549.

Larson, P. D., \& Gammelgaard, B. (2001). The logistics triad: Survey and case study results. Transportation Journal.

Lieb, R., \& Randall, H. (1999). 1997 CEO perspectives on the current status and future prospects of the third party logistics industry in the united states. Transportation Journal, 28.

Maloni, M. J., \& Carter, C. R. (2006). Opportunities for Research in Third-Party Logistics. Transportation Journal.

McCarthy, T., \& Golicic, S. (2002). Implementing collaborative forecasting to improve supply chain performance. International Journal of Physical Distribution \& Logistics Management, 431.

Mentzer, J. (2005). Sales Forecasting Management: A Demand Management Approach. SAGE Publications.

Morash, E. A. (2001). Supply chain strategies, capabilities, and performance. Transportation Journal.

Mukhopadhyay, S. K., \& Setaputra, R. (2006). The role of $4 \mathrm{PL}$ as the reverse logistics integrator Optimal pricing and return policies. The International Journal of Logistics Management.

Parkhe, A. (1993). Strategic Alliance Structuring: A Game Theoretic And Transaction Cost Examination Of Interfirm Cooperation. Intereconomics. 
Pfohl, H. C., \& Buse, H. P. (2000). Inter-organizational logistics systems in flexible production networks An organizational capabilities perspective. International Journal of Physical Distribution \& Logistics Management.

Power, D., Sharafali, M., \& Bhakoo, V. (2007). Adding value through outsourcing contribution of $3 \mathrm{pl}$ services to customer performance. Management Research News, 228-235.

Ring, P. S., \& Van De Ven, A. H. (1992). Structuring Cooperative Relationships Between Organizations. Strategic Management Journal.

Ring, P. S., \& Van De Ven, A. H. (1994). Developmental processes of cooperative interorganizational relationships. Academy of Management. The Academy of Management Review.

Simatupang, T. M. (2004). Applying the theory of constraints to supply chain collaboration. Supply Chain Management: An International Journal, 57-70.

Simatupang, T. M., \& Sridharan, R. (2004). Benchmarking supply chain collaboration: An empirical study. Benchmarking: An international Journal, 484-503.

Simatupang, T. M., \& Sridharan, R. (2005). The collaboration index: a measure for supply chain collaboration. International Journal of Physical Distribution \& Logistics Management.

Simatupang, T. M., Wright, A. C., \& Sridharan, R. (2002). The knowledge of coordination for supply chain integration. Business Process Management Journal.

Smith R., P., \& Van De Ven, A. H. (1992). Structuring Cooperative Relationships Between Organizations. Strategic Management Journal.
Spiegel, Y. (1993). Horizontal subcontracting. The Rand Journal of Economics.

Stank, T. P., Keller, S. B., \& Closs, D. J. (2001). Transportation Journal. Transportation Journal.

Stefansson, G. (2006). Collaborative logistics management and the role of third-party service providers. International Journal of Physical Distribution \& Logistics Management, 76-92.

Todeva, E., \& Knoke, D. (2005). Strategic alliances and models of collaboration. Management Decision, 123-148.

Vogelaar, F. (2002). Modernisation of Ec competition law, economy and horizontal cooperation between Undertakings Modernisation of EC competition law, economy and horizontal cooperation between Undertakings. Intereconomics.

Wang, Y. C., \& Ho, S. C. (2006). Information Systems Dispatching in the Global Environment, Acer, A Case of Horizontal inegration. Journal of Cases on Information Technology.

Whipple, J. M., \& Frankel, R. (2000). Strategic alliance success factors. Journal of Supply Chain Management.

Zailani, S., \& Rajagopal, P. (2005). Supply chain integration and performance: US versus East Asian companies. Supply Chain Management.

Zineldin, M. (2004). Co-Opetition: The organization of the Future. Marketing Intelligence \& Planning, 780.

Zineldin, M., \& Bredenlow, T. (2003). Strategic alliance: Synergies and challenges: A case of strategic outsourcing relationship "sour". International Journal of Physical Distribution \& Logistics Management. 